Pacific

Journal of

Mathematics

DIMENSION GROUPS OF TOPOLOGICAL JOININGS AND NON-COALESCENCE OF CANTOR MINIMAL SYSTEMS

Hiroki Matui

Volume $204 \quad$ No. 1

May 2002 


\title{
DIMENSION GROUPS OF TOPOLOGICAL JOININGS AND NON-COALESCENCE OF CANTOR MINIMAL SYSTEMS
}

\author{
HiRoki Matui
}

\begin{abstract}
When we have two extensions of a Cantor minimal system which are both one-to-one on at least one orbit, we can construct new Cantor minimal systems called topological joinings. We compute the dimension group of the joining in a special case. As an application, we show that a non-invertible endomorphism can induce the identity map on the dimension group of a Cantor minimal system.
\end{abstract}

\section{Introduction.}

By a topological dynamical system $(Y, \psi)$, we mean a compact Hausdorff space $Y$ endowed with a homeomorphism $\psi$. When $\left(Y_{i}, \psi_{i}\right), i=0,1$ are two topological dynamical systems, $\psi_{0} \times \psi_{1}$-invariant closed subsets of $Y_{0} \times Y_{1}$ are called (topological) joinings, and when $\left(Y_{0}, \psi_{0}\right)$ equals $\left(Y_{1}, \psi_{1}\right)$, they are called self-joinings. In the measure-theoretical setting, the notion of selfjoinings was introduced by D. Rudolph in $[\mathbf{R}]$, and it was proved that the minimal self-joining property implies coalescence and zero entropy. In this paper, we will compute the dimension group of joinings of Cantor minimal systems.

When $Y$ is the Cantor set and a homeomorphism $\psi$ on $Y$ has no nontrivial invariant closed set, $(Y, \psi)$ is called a Cantor minimal system. We define the dimension group $K^{0}(Y, \psi)$ of $(Y, \psi)$ as the quotient of $C(Y, \mathbf{Z})$ by the coboundary subgroup

$$
B_{\psi}=\left\{f-f \circ \psi^{-1} ; f \in C(Y, \mathbf{Z})\right\} .
$$

In [GPS], it was proved that the dimension group $K^{0}(Y, \psi)$, as an ordered group with a distinguished order unit, characterizes the strong orbit equivalence class of $(Y, \psi)$.

We would like to consider the case that a joining $(Z, \tau)$ of Cantor minimal systems $\left(Y_{0}, \psi_{0}\right)$ and $\left(Y_{1}, \psi_{1}\right)$ is also a Cantor minimal system. (We must distinguish the property of minimal self-joinings and minimal systems in the joinings.) We don't know a necessary and sufficient condition so that the joining is minimal. In a special case, however, we will prove that the joining becomes a Cantor minimal system, and compute its dimension group. Our 
main result is Theorem 5, in which we will show that the dimension group of the joining is order isomorphic to the relative direct sum of the $K^{0}\left(Y_{i}, \psi_{i}\right)$ 's.

In the last section, we will consider the non-coalescence of Cantor minimal systems. We denote by $C(\psi)$ the set of continuous maps on $Y$ which commute with $\psi$ and call it the centralizer of $(Y, \psi)$. If $C(\psi)$ consists of homeomorphisms, we say that the system $(Y, \psi)$ is topologically coalescent, and say that $(Y, \psi)$ is non-coalescent, if $C(\psi)$ contains a non-invertible endomorphism. For an element $\gamma \in C(\psi)$, we can define an order homomorphism $\bmod (\gamma)$ on $K^{0}(Y, \psi)$ by $\bmod (\gamma)([f])=[f \circ \gamma]$ for $[f] \in K^{0}(Y, \psi)$. The map mod gives a homomorphism from the automorphism group of $(Y, \psi)$ to the automorphism group of the dimension group. Finite subgroups of the kernel of the mod map were studied by the author in [M2]. In the present paper, we will prove that there exist a lot of non-coalescent Cantor minimal systems whose non-invertible endomorphisms induce automorphisms on the dimension groups. Especially, we construct a non-coalescent Cantor minimal system which is strong orbit equivalent to an odometer system. For detailed information on odometer groups, we refer to $[\mathbf{H R}]$.

\section{Dimension groups of joinings.}

Let $\left\{\left(Y_{i}, \psi_{i}\right)\right\}_{i \in I}$ be a family of topological dynamical systems. For $\left(y_{i}\right)_{i \in I}$ in $Y^{I}$, the orbit closure of $\left(y_{i}\right)_{i}$ by the diagonal action is called the joining generated by $\left(y_{i}\right)_{i}$.

Lemma 1. Let $\left\{\left(Y_{i}, \psi_{i}\right)\right\}_{i \in I}$ and $(X, \phi)$ be topological dynamical systems and $\pi_{i}:\left(Y_{i}, \psi_{i}\right) \rightarrow(X, \phi)$ be factor maps. If $y=\left(y_{i}\right)_{i \in I}$ satisfies $\pi_{i}\left(y_{i}\right)=$ $\pi_{j}\left(y_{j}\right)$ and $\pi_{i}^{-1}\left(\pi_{i}\left(y_{i}\right)\right)=\left\{y_{i}\right\}$ for all $i, j \in I$, and $(X, \phi)$ is minimal, then the joining generated by $\left(y_{i}\right)_{i \in I} \in Y^{I}$ is minimal.

Proof. It suffices to prove the case $I=\{0,1\}$. Let $\psi$ be the diagonal action $\psi_{0} \times \psi_{1}$. For every open neighborhood $O$ of $y=\left(y_{0}, y_{1}\right)$, there exists an open set $U$ of $X$ such that $V=\pi_{0}^{-1}(U) \times \pi_{1}^{-1}(U)$ is a neighborhood of $y$ contained in $O$. To prove the minimality, it is enough to show the almost periodicity of $y$ for $V$, that is,

$$
\left\{k \in \mathbf{Z} ; \psi^{k}(y) \in V\right\}
$$

has a bounded gap in Z Z. But, we have $\psi^{k}(y) \in V$ if and only if $\phi^{k}\left(\pi_{0}\left(y_{0}\right)\right) \in$ $U$, and so the assertion comes from the minimality of $(X, \phi)$.

When an unperforated ordered group $G$ satisfies the Riesz interpolation property, $G$ is called a dimension group in the abstract sense ([GPS, Section $1]$ ). Let $\pi: H \rightarrow G$ be an order homomorphism between dimension groups. We say that $\pi$ is an order embedding, if $\pi$ is injective and $\pi(h) \geq 0$ if and only if $h \geq 0$. 
Definition 2. Let $\pi_{i}: H \rightarrow G_{i}, i=0,1$, be order embeddings between simple dimension groups, none of them equal to $\mathbf{Z}$. Assuming $G_{i} / \pi_{i}(H)$ is torsion-free for $i=0,1$, we call the quotient $D$ of $G_{0} \oplus G_{1}$ by

$$
\left\{\left(\pi_{0}(h),-\pi_{1}(h)\right) ; h \in H\right\}
$$

the relative direct sum of $G_{i}$ with respect to $\pi_{i}(H)$. Define the positive cone $D^{+}$such as

$$
D^{+}=\left\{\left[g_{0}, g_{1}\right] \in D ; g_{i} \in G_{i}^{+}, i=0,1\right\},
$$

where $[\cdot, \cdot]$ means the quotient map. When $\pi_{0}$ and $\pi_{1}$ preserve the distinguished order units, the order unit of $\left(D, D^{+}\right)$can be defined in the obvious way.

We can define the relative direct sum of more than two dimension groups in a similar fashion.

Lemma 3. Let $\left(D, D^{+}\right)$be the relative direct sum of $G_{i}$ with respect to $\pi_{i}(H)$. Then, $\left(D, D^{+}\right)$is an unperforated ordered group, and for $x \in D^{+} \backslash$ $\{0\}$ and $y \in D^{+}$, there exists a natural number $n \in \mathbf{N}$ such that $0 \leq y \leq n x$.

Proof. It is easy to see that $\left(D, D^{+}\right)$is an ordered group. Suppose $n\left[g_{0}, g_{1}\right] \geq$ 0 for $\left[g_{0}, g_{1}\right] \in D$ and $n \in \mathbf{N}$. By the definition, there exists an element $h \in H$ such that $n g_{0}+\pi_{0}(h) \geq 0$ and $n g_{1}-\pi_{1}(h) \geq 0$. If $n g_{0}+\pi_{0}(h)$ is equal to zero, $h$ is divisible by $n$ in $H$, because $G_{0} / \pi_{0}(H)$ is torsionfree. Hence, we get $\left[g_{0}, g_{1}\right] \geq 0$ in $D$. We have the same conclusion, if $n g_{1}-\pi_{1}(h)$ is zero. Therefore, we may assume the strict inequality. Since $H$ is a simple dimension group $(\neq \mathbf{Z})$, we can find $\epsilon_{i} \in H$ for $i=0,1$ such that $0<\pi_{0}\left(\epsilon_{0}\right)<n g_{0}+\pi_{0}(h)$ and $0<\pi_{1}\left(\epsilon_{1}\right)<n g_{1}-\pi_{1}(h)$, by Corollary 4.10 of $[\mathbf{G H}]$. By using 4.10 of $[\mathbf{G H}]$ again, we get an element $h^{\prime}$ such that

$$
h-\epsilon_{0}<n h^{\prime}<h+\epsilon_{1} .
$$

Then, $g_{0}+\pi_{0}\left(h^{\prime}\right)$ and $g_{1}-\pi_{1}\left(h^{\prime}\right)$ are positive, which implies $\left[g_{0}, g_{1}\right]$ are positive in $D$.

To prove the second statement, let $x=\left[g_{0}, g_{1}\right]$ be in $D^{+} \backslash\{0\}$. We may assume $g_{0}>0$ and $g_{1} \geq 0$. Because we can choose $h \in H^{+} \backslash\{0\}$ such that $g_{0}-\pi_{0}(h)>0$, the assertion has been proved.

The relative direct sum $D$ may not satisfy the Riesz interpolation property. For example, let $G$ be $\mathbf{Q}^{2}$ and define the positive cone by

$$
G^{+}=\{(x, y) ; x>|y|\} \cup\{(0,0)\} .
$$

If $H=\{(x, 0)\} \cong \mathbf{Q}$ is a subgroup of $G$, it can be seen that the relative direct sum of two copies of $G$ with respect to $H$ doesn't satisfy the Riesz interpolation property.

We say $\pi(H)$ is order dense in $G$, if there is $h \in H$ for every $g<g^{\prime}$ such that $g<\pi(h)<g^{\prime}$. By [GPS2, Proposition 1.1], $\pi(H)$ is order dense in $G$, 
if and only if $\pi$ induces an isomorphism between the state space $S(G)$ and $S(H)$.

Lemma 4. The relative direct sum $\left(D, D^{+}\right)$of $G_{i}$ with respect to $\pi_{i}(H)$ satisfies the Riesz interpolation property, if $\pi_{1}(H)$ is order dense in $G_{1}$.

Proof. We check the Riesz decomposition property. Assume the inequality $0 \leq\left[a, a^{\prime}\right]<\left[b, b^{\prime}\right]+\left[c, c^{\prime}\right]$ in $D$ for $a, b, c \in G_{0}^{+}$and $a^{\prime}, b^{\prime}, c^{\prime} \in G_{1}^{+}$. We must show that there exist $x, y \in D^{+}$so that $\left[a, a^{\prime}\right]=x+y, x \leq\left[b, b^{\prime}\right], y \leq\left[c, c^{\prime}\right]$. We may also assume $\left[b, b^{\prime}\right] \neq 0$ and $\left[c, c^{\prime}\right] \neq 0$. Because $\left[b+c, b^{\prime}+c^{\prime}\right]$ is strictly larger than $\left[a, a^{\prime}\right]$, we can find $v, \epsilon \in H$ such that

$$
0 \leq(b+c)-a+\pi_{0}(v), \quad 0<2 \pi_{1}(\epsilon)<\left(b^{\prime}+c^{\prime}\right)-a^{\prime}-\pi_{1}(v) .
$$

Since $\pi_{1}(H)$ is order dense in $G_{1}$, we also get $t, u \in H^{+}$satisfying,

$$
0 \leq b+\pi_{0}(t), \quad 0<b^{\prime}-\pi_{1}(t)<\pi_{1}(\epsilon)
$$

and

$$
0 \leq c+\pi_{0}(u), \quad 0<c^{\prime}-\pi_{1}(u)<\pi_{1}(\epsilon) .
$$

Take $\epsilon^{\prime} \in H$ such as $0<\pi_{1}\left(\epsilon^{\prime}\right)<b^{\prime}-\pi_{1}(t)$ and $0<\pi_{1}\left(\epsilon^{\prime}\right)<c^{\prime}-\pi_{1}(u)$. By using the order density again, we have $s \in H^{+}$satisfying

$$
0 \leq a+\pi_{0}(s), \quad 0<a^{\prime}-\pi_{1}(s)<\pi_{1}\left(\epsilon^{\prime}\right) .
$$

Then, we obtain

$$
\pi_{1}\left(\epsilon^{\prime}\right)<\left(b^{\prime}+c^{\prime}\right)-a^{\prime}-\pi_{1}(t+u-s)<2 \pi_{1}(\epsilon),
$$

and so $t+u-s$ is greater than $v$. If we replace $\left[a, a^{\prime}\right],\left[b, b^{\prime}\right]$ and $\left[c, c^{\prime}\right]$ with $\left[a+\pi_{0}(s), a^{\prime}-\pi_{1}(s)\right],\left[b+\pi_{0}(t), b^{\prime}-\pi_{1}(t)\right]$ and $\left[c+\pi_{0}(u), c^{\prime}-\pi_{1}(u)\right]$, the assertion follows by the Riesz decomposition property of $G_{i}$.

We would like to state the main theorem. Let $\pi_{i}:\left(Y_{i}, \psi_{i}\right) \rightarrow(X, \phi)$ be factor maps between Cantor minimal systems for $i=0,1$. Define

$$
E_{i}=\left\{x \in X ; \# \pi_{i}^{-1}(x) \neq 1\right\}
$$

for each $i$ and assume that $y_{i, \max } \in Y_{i}$ and $x_{\max } \in X$ satisfy

$$
\pi_{0}\left(y_{0, \max }\right)=\pi_{1}\left(y_{1, \max }\right)=x_{\max } \in\left(E_{0} \cup E_{1}\right)^{c} .
$$

By Lemma 1 , the joining $(Z, \tau)$ generated by $\left(y_{0, \max }, y_{1, \max }\right)$ is a Cantor minimal system. In general, a factor map $\pi_{i}$ between Cantor minimal systems induces the order embedding $\pi_{i}^{*}: K^{0}(X, \phi) \rightarrow K^{0}\left(Y_{i}, \psi_{i}\right)$. In Lemma 6, we will prove that the quotient of $K^{0}\left(Y_{i}, \psi_{i}\right)$ by $\pi_{i}^{*}\left(K^{0}(X, \phi)\right)$ is torsionfree. We denote by $p_{i}$ the canonical factor maps from $(Z, \tau)$ to $\left(Y_{i}, \psi_{i}\right)$ for $i=0,1$. The direct sum of $p_{0}^{*}$ and $p_{1}^{*}$ induces the homomorphism from $K^{0}\left(Y_{0}, \psi_{0}\right) \oplus K^{0}\left(Y_{1}, \psi_{1}\right)$ to $K^{0}(Z, \tau)$. 
Theorem 5. In the above setting, we further assume that $E_{0}$ and $E_{1}$ are disjoint. Then, the dimension group $K^{0}(Z, \tau)$ is isomorphic to the relative direct sum of $K^{0}\left(Y_{i}, \psi_{i}\right)$ with respect to $\pi_{i}\left(K^{0}(X, \phi)\right)$ as a unital ordered group. Moreover, the state space of $K^{0}(Z, \tau)$ is equal to

$$
\left\{\left(\mu_{0}, \mu_{1}\right) ; \mu_{i} \in S\left(K^{0}\left(Y_{i}, \psi_{i}\right)\right), \mu_{0} \circ \pi_{0}^{*}=\mu_{1} \circ \pi_{1}^{*}\right\},
$$

where $S\left(K^{0}\left(Y_{i}, \psi_{i}\right)\right)$ is the state space of $K^{0}\left(Y_{i}, \psi_{i}\right)$.

If $E_{0}$ and $E_{1}$ are disjoint, the topological joining $Z$ coincides with the pull-back associated with $\pi_{0}$ and $\pi_{1}$, that is,

$$
Z=\left\{\left(y_{0}, y_{1}\right) \in Y_{0} \times Y_{1} ; \pi_{0}\left(y_{0}\right)=\pi_{1}\left(y_{1}\right)\right\} .
$$

Therefore, the theorem asserts that the dimension group of the pull-back system is isomorphic to the push-out of the dimension group.

For an ergodic measure $\mu$ on $(X, \phi)$, either of $\mu\left(E_{0}\right)$ and $\mu\left(E_{1}\right)$ must be zero when $E_{0}$ and $E_{1}$ are disjoint. If $\mu\left(E_{0}\right)=0$ for every ergodic measure $\mu$, then $\pi_{0}^{*}\left(K^{0}(X, \phi)\right)$ is order dense in $K^{0}\left(Y_{0}, \psi_{0}\right)$. But, we must remark that even if $E_{0}$ and $E_{1}$ are disjoint, $\pi_{i}^{*}\left(K^{0}(X, \phi)\right)$ may not be order dense in $K^{0}\left(Y_{i}, \psi_{i}\right)$ for each $i=0,1$. See the example at the last of this section.

In order to prove the above theorem, at first, we need to fix KakutaniRohlin partitions for $(X, \phi)$ and $\left(Y_{i}, \psi_{i}\right)$.

Let $\left\{X_{n}\right\}_{n \in \mathbf{N}}$ be a decreasing sequence of clopen sets of $X$ such that the intersection of all $X_{n}$ 's is $\left\{x_{\max }\right\}$. By Theorem 4.2 of [HPS], for each $X_{n}$, we can find a finite set $V_{n}$, a map $h: V_{n} \rightarrow \mathbf{N}$ and a clopen partition

$$
\mathcal{P}_{n}=\left\{X(n, v, k) ; v \in V_{n}, 1 \leq k \leq h(v)\right\}
$$

of $X$, which satisfy the following properties:

- For every $v \in V_{n}$ and $k \neq h(v)$, we have $\phi(X(n, v, k))=X(n, v, k+1)$.

- The clopen set $X_{n}$ equals the disjoint union of $X(n, v, h(v))$ for all $v \in V_{n}$.

- The partition $\mathcal{P}_{n+1}$ is finer than $\mathcal{P}_{n}$ for every $n \in \mathbf{N}$, and the family of partitions $\left\{\mathcal{P}_{n}\right\}_{n}$ generates the topology of $X$.

The clopen set $X_{n}$ is called the top set and the map $h$ is called the height function. In order to compute the dimension group $K^{0}(X, \phi)$, we must consider the free abelian group $\mathbf{Z}^{V_{n}}$. For convenience we denote the canonical basis of $\mathbf{Z}^{V_{n}}$ by the vertices $v_{1}, v_{2}, \cdots, v_{j} \in V_{n}$. When we define the rectangular matrix $A_{n} \in M_{V_{n} \times V_{n+1}}(\mathbf{Z})$ by

$$
A_{n}\left(v, v^{\prime}\right)=\#\left\{1 \leq k \leq h\left(v^{\prime}\right) ; X\left(n+1, v^{\prime}, k\right) \subset X(n, v, h(v))\right\}
$$

for every $v \in V_{n}$ and $v^{\prime} \in V_{n+1}$, the dimension group $K^{0}(X, \phi)$ is computed by the inductive limit of $\left(\mathbf{Z}^{V_{n}}\right)_{n}$ with the connecting maps $\left(A_{n}\right)_{n}$.

The partition $\mathcal{P}_{n}$ induces the clopen partition of $Y_{i}$ for each $i=0,1$ and $n \in \mathbf{N}$. This family of partitions, however, does not generate the topology of $Y_{i}$. When we divide every clopen set $\pi_{i}^{-1}(X(n, v, k))$ so that the topology 
is generated, we obtain a finite set $W_{i, n}$, a map $\rho_{i}: W_{i, n} \rightarrow V_{n}$ and a clopen partition

$$
\mathcal{Q}_{i, n}=\left\{Y_{i}(n, w, k) ; w \in W_{i, n}, 1 \leq k \leq h\left(\rho_{i}(w)\right)\right\}
$$

of $Y_{i}$ and the following holds for each $i=0,1$ :

- For every $w \in W_{i, n}$ and $k \neq h\left(\rho_{i}(w)\right)$, we have $\psi_{i}\left(Y_{i}(n, w, k)\right)=$ $Y_{i}(n, w, k+1)$.

- For every $v \in V_{n}$ and $1 \leq k \leq h(v), \pi_{i}^{-1}(X(n, v, k))$ is the disjoint union of $Y_{i}(n, w, k)$ for all $w \in \rho_{i}^{-1}(v)$.

- As in the case of $(X, \phi), Y_{i, n}=\pi_{i}^{-1}\left(X_{n}\right)$ is the disjoint union of $Y_{i}\left(n, w, h\left(\rho_{i}(w)\right)\right)$ for all $w \in W_{i, n}$.

- The partition $\mathcal{Q}_{i, n+1}$ is finer than $\mathcal{Q}_{i, n}$ and the family of partitions $\left\{\mathcal{Q}_{i, n}\right\}_{n}$ generates the topology of $Y_{i}$.

Notice that the intersection of all $Y_{i, n}$ 's equals $\left\{y_{i, \max }\right\}$ because $x_{\max }$ is not contained in $E_{i}$. As above we denote the canonical basis of $\mathbf{Z}^{W_{i, n}}$ by the vertices $w$ in $W_{i . n}$. We can compute the dimension group $K^{0}\left(Y_{i}, \psi_{i}\right)$, using the rectangular matrix $B_{i, n} \in M_{W_{i, n} \times W_{i, n+1}}(\mathbf{Z})$ determined in a similar fashion to the case of $A_{n}$. The homomorphisms sending $v \in \mathbf{Z}^{V_{n}}$ to $\sum_{w \in \rho_{i}^{-1}(v)} w \in$ $\mathbf{Z}^{W_{i, n}}$ induce the order embedding $\pi_{i}^{*}: K^{0}(X, \phi) \rightarrow K^{0}\left(Y_{i}, \psi_{i}\right)$.

Lemma 6. Let $\pi:(Y, \psi) \rightarrow(X, \phi)$ be a factor map between Cantor minimal systems. If there is a point $y \in Y$ such that $\pi^{-1}(\pi(y))=\{y\}$, then the quotient $K^{0}(Y, \psi) / \pi^{*}\left(K^{0}(X, \phi)\right)$ is torsion-free.

Proof. We will prove the lemma for $\pi_{0}:\left(Y_{0}, \psi_{0}\right) \rightarrow(X, \phi)$ above. The embedding of $K^{0}(X, \phi)$ to $K^{0}\left(Y_{0}, \psi_{0}\right)$ is obtained by the natural injection from $\mathbf{Z}^{V_{n}}$ to $\mathbf{Z}^{W_{0, n}}$ induced by $\rho_{0}$. Since clearly $\mathbf{Z}^{W_{0, n}} / \mathbf{Z}^{V_{n}}$ is torsion-free, we get the conclusion.

We return to the setting of Theorem 5. We would like to describe Kakutani-Rohlin partitions of $(Z, \tau)$. When $w_{0} \in W_{0, n}$ and $w_{1} \in W_{1, n}$ are preimages of the same $v \in V_{n}$, we set

$$
Z\left(n, w_{0}, w_{1}, k\right)=Z \cap\left(Y_{0}\left(n, w_{0}, k\right) \times Y_{1}\left(n, w_{1}, k\right)\right)
$$

for all $1 \leq k \leq h(v)$. Let $W_{n}$ be the set of pairs $\left(w_{0}, w_{1}\right)$ such that the clopen set $Z\left(n, w_{0}, w_{1}, h(v)\right)$ is not empty. Then, we get the partition

$$
\mathcal{R}_{n}=\left\{Z\left(n, w_{0}, w_{1}, k\right) ;\left(w_{0}, w_{1}\right) \in W_{n}, 1 \leq k \leq h\left(\rho_{i}\left(w_{i}\right)\right)\right\}
$$

of $Z$. We can check that the sequence of clopen partitions $\left\{\mathcal{R}_{n}\right\}_{n}$ satisfies the similar properties as in the case of $\left\{\mathcal{P}_{n}\right\}_{n}$ or $\left\{\mathcal{Q}_{i, n}\right\}_{n}$. Hence, we can compute the dimension group $K^{0}(Z, \tau)$ by the inductive limit of $\left(\mathbf{Z}^{W_{n}}\right)_{n}$. We denote the canonical basis of $\mathbf{Z}^{W_{n}}$ by the vertices $\left(w_{0}, w_{1}\right)$ in $W_{n}$. The connecting map from $\mathbf{Z}^{W_{n}}$ to $\mathbf{Z}^{W_{n+1}}$ is given by a matrix $C_{n}$ and the entry 
of $C_{n}$ corresponding to $\left(w_{0}, w_{1}\right) \in W_{n}$ and $\left(w_{0}^{\prime}, w_{1}^{\prime}\right) \in W_{n+1}$ is equal to the cardinality of the set

$$
\left\{1 \leq k \leq h\left(\rho_{i}\left(w_{i}^{\prime}\right)\right) ; Z\left(n, w_{0}^{\prime}, w_{1}^{\prime}, k\right) \subset Z\left(n, w_{0}, w_{1}, h\left(\rho_{i}\left(w_{i}\right)\right)\right)\right\} .
$$

The order embedding $p_{0}^{*}$ is induced from the homomorphism sending $w_{0} \in$ $\mathbf{Z}^{W_{0, n}}$ to $\sum_{\left(w_{0}, w\right) \in W_{n}}\left(w_{0}, w\right) \in \mathbf{Z}^{W_{n}}$. The order embedding $p_{1}^{*}$ is described in the same way.

Define

$$
E_{i}(m, Q)=\left\{P \in \mathcal{P}_{m} ; \pi_{i}^{-1}(P) \cap Q \text { and } \pi_{i}^{-1}(P) \cap Q^{c} \text { are both nonempty }\right\}
$$

for $Q \in \mathcal{Q}_{i, n}, n \leq m$ and $i=0,1$. If $P^{\prime} \in \mathcal{P}_{m+1}$ is a subset of $P \in \mathcal{P}_{m}$ and $P^{\prime} \in E_{i}(m+1, Q)$, then we have $P \in E_{i}(m, Q)$.

Lemma 7. For every $Q_{0} \in \mathcal{Q}_{0, n}$ and $Q_{1} \in \mathcal{Q}_{1, n}$, there exists a natural number $m$ greater than $n$ such that $E_{0}\left(m, Q_{0}\right)$ and $E_{1}\left(m, Q_{1}\right)$ are disjoint.

Proof. The proof is by contradiction. Let $U_{i, m}$ be the disjoint union of $P \in$ $E_{i}\left(m, Q_{i}\right)$, for every $m \geq n$ and $i=0,1$. The clopen set $U_{m}=U_{0, m} \cap U_{1, m}$ is nonempty and forms a decreasing sequence. Therefore, there exists a point $x \in X$ contained in all $U_{m}$. Then, $\pi_{i}^{-1}(x)$ contains at least two distinct points for each $i=0,1$, which contradicts the disjointness of $E_{0}$ and $E_{1}$.

We are now ready to prove the main theorem.

Proof of Theorem 5. By virtue of Lemma 7, we may assume that $E_{0}(n+$ $\left.1, Q_{0}\right)$ and $E_{1}\left(n+1, Q_{1}\right)$ are disjoint for all $Q_{i} \in \mathcal{Q}_{i, n}$ and $n \in \mathbf{N}$. We denote the factor map $\pi_{0} \circ p_{0}=\pi_{1} \circ p_{1}$ by $\pi$.

At first, we prove the surjectivity of the map $p_{0}^{*} \oplus p_{1}^{*}$ from $K^{0}\left(Y_{0}, \psi_{0}\right) \oplus$ $K^{0}\left(Y_{1}, \psi_{1}\right)$ to $K^{0}(Z, \tau)$. Fix $n \in \mathbf{N}$ and $\left(w_{0}, w_{1}\right) \in W_{n}$ arbitrarily. Let $v=\rho_{0}\left(w_{0}\right)=\rho_{1}\left(w_{1}\right) \in V_{n}$. Set

$$
\begin{gathered}
\mathcal{S}=\left\{R \in \mathcal{R}_{n+1} ; R \cap Z\left(n, w_{0}, w_{1}, h(v)\right) \text { is not empty }\right\}, \\
\mathcal{S}_{i}=\left\{R \in \mathcal{S} ; R \subset \pi^{-1}(P) \text { for some } P \in E_{i}\left(n+1, Y_{i}\left(n, w_{i}, h(v)\right)\right)\right\},
\end{gathered}
$$

for each $i=0,1$, and

$$
\begin{aligned}
\mathcal{S}_{2}=\{R \in \mathcal{S} ; & R \cap \pi^{-1}(P) \\
& \text { is empty for all } \left.P \in E_{i}\left(n+1, Y_{i}\left(n, w_{i}, h(v)\right)\right), i=0,1\right\} .
\end{aligned}
$$

Then, we have

$$
\begin{aligned}
Z\left(n, w_{0}, w_{1}, h(v)\right) & =\bigcup_{R \in \mathcal{S}} R \\
& =\bigcup_{R \in \mathcal{S}_{0}} R \cup \bigcup_{R \in \mathcal{S}_{1}} R \cup \bigcup_{R \in \mathcal{S}_{2}} R .
\end{aligned}
$$


The first summand is equal to

$$
\bigcup Z \cap\left(Y_{0}\left(n+1, w_{0}^{\prime}, k\right) \times Y_{1}\right),
$$

where the union runs over $w_{0}^{\prime}$ and $k$ satisfying $Z\left(n+1, w_{0}^{\prime}, w_{1}^{\prime}, k\right) \in \mathcal{S}_{0}$ for some $w_{1}^{\prime} \in W_{1, n+1}$. The second summand can be written in the same way. The last summand is equal to $\pi^{-1}(U)$ for some clopen set $U$ of $X$. Hence, the surjectivity has been proved.

Let us consider the kernel of $p_{0}^{*} \oplus p_{1}^{*}$. Suppose $a_{0}=\sum_{w_{0} \in W_{0, n}} \lambda_{0, w_{0}} w_{0}$ and $a_{1}=\sum_{w_{1} \in W_{1, n}} \lambda_{1, w_{1}} w_{1}$ satisfy

$$
p_{0}^{*}\left(a_{0}\right)=p_{1}^{*}\left(a_{1}\right)
$$

in $K^{0}(Z, \tau)$. We may assume that

$$
\sum_{\left(w_{0}, w_{1}\right) \in W_{n}} \lambda_{0, w_{0}}\left(w_{0}, w_{1}\right)=\sum_{\left(w_{0}, w_{1}\right) \in W_{n}} \lambda_{1, w_{1}}\left(w_{0}, w_{1}\right),
$$

and so we have $\lambda_{0, w_{0}}=\lambda_{1, w_{1}}$, if $\left(w_{0}, w_{1}\right)$ exists in $W_{n}$. Therefore, we may further assume that there exists a subset $F_{i} \subset W_{i, n}$ for each $i=0,1$, such that:

- If $w_{0} \in F_{0}$ and $\left(w_{0}, w_{1}\right) \in W_{n}$, then $w_{1} \in F_{1}$.

- If $w_{1} \in F_{1}$ and $\left(w_{0}, w_{1}\right) \in W_{n}$, then $w_{0} \in F_{0}$.

- The element $a_{i}$ is equal to $\sum_{w_{i} \in F_{i}}\left(w_{0}, w_{1}\right)$ for each $i=0,1$.

Define the clopen set

$$
W=\bigcup_{w_{0} \in F_{0}} Z\left(n, w_{0}, w_{1}, h\left(\rho_{i}\left(w_{i}\right)\right)\right)=\bigcup_{w_{1} \in F_{1}} Z\left(n, w_{0}, w_{1}, h\left(\rho_{i}\left(w_{i}\right)\right)\right) .
$$

Let $Z\left(n+1, w_{0}^{\prime}, w_{1}^{\prime}, k\right) \in \mathcal{R}_{n+1}$ be a clopen set which is contained in $W$. Then, there exist $w_{0} \in F_{0}$ and $w_{1} \in F_{1}$ such that $Z\left(n+1, w_{0}^{\prime}, w_{1}^{\prime}, k\right)$ is contained in $Z\left(n, w_{0}, w_{1}, h\left(\rho_{i}\left(w_{i}\right)\right)\right)$. Without loss of generality, we may assume that $X\left(n+1, \rho_{0}\left(w_{0}^{\prime}\right), k\right)$ is not contained in $E_{0}\left(n+1, Y_{0}\left(n, w_{0}, h\left(\rho_{0}\left(w_{0}\right)\right)\right)\right)$. Take a pair $\left(w_{0}^{\prime \prime}, w_{1}^{\prime \prime}\right) \in W_{n+1}$ satisfying $\rho_{i}\left(w_{i}^{\prime \prime}\right)=\rho_{i}\left(w_{i}^{\prime}\right)$. There exists a pair $\left(\widetilde{w_{0}}, \widetilde{w_{1}}\right) \in W_{n}$ satisfying $Z\left(n+1, w_{0}^{\prime \prime}, w_{1}^{\prime \prime}, k\right) \subset Z\left(n, \widetilde{w_{0}}, \widetilde{w_{1}}, h\left(\rho_{i}\left(\widetilde{w_{i}}\right)\right)\right)$. By the assumption, we have $\widetilde{w_{0}}=w_{0}$, and so $\widetilde{w_{1}}$ must be in $F_{1}$. Hence, $\pi^{-1}\left(X\left(n+1, \rho_{0}\left(w_{0}^{\prime}\right), k\right)\right)$ is contained in $W$. By repeating this argument, we see that $p_{0}^{*}\left(a_{0}\right)=p_{1}^{*}\left(a_{1}\right)$ comes from $K^{0}(X, \phi)$.

We have proved that $p_{0}^{*} \oplus p_{1}^{*}$ induces an isomorphism from the relative direct sum to $K^{0}(Z, \tau)$. It is obvious from the above argument that the isomorphism preserves the positive cones and the distinguished order units, and so the proof is completed.

Corollary 8. Let $\left\{\left(Y_{i}, \psi_{i}\right)\right\}_{i \in I}$ be a family of at most countable Cantor minimal systems and $\pi_{i}:\left(Y_{i}, \psi_{i}\right) \rightarrow(X, \phi)$ be factor maps to a Cantor minimal system $(X, \phi)$. Define

$$
E_{i}=\left\{x \in X ; \# \pi_{i}^{-1}(x) \neq 1\right\}
$$


and assume $y_{i} \in Y_{i}$ and $x \in X$ satisfies $\pi_{i}\left(y_{i}\right)=x$ and $x \in E_{i}^{c}$ for all $i \in I$. When we further assume that $E_{i}$ 's are disjoint for all $i$, the dimension group of the joining $(Z, \tau)$ generated by $\left(y_{i}\right)_{i}$ is isomorphic to the relative direct sum of $K^{0}\left(Y_{i}, \psi_{i}\right)$ 's with respect to $\pi_{i}^{*}\left(K^{0}(X, \phi)\right)$.

Proof. Use Theorem 5 repeatedly.

Example. Let $\left\{q_{n}\right\}_{n \in \mathbf{N}}$ be a sequence of natural numbers satisfying

$$
\alpha=\lim _{n \rightarrow \infty} \prod_{k=1}^{n} \frac{q_{k}+1}{q_{k}-1}<\infty .
$$

For convenience, we assume that, for every $p \in \mathbf{N}$, there exists $n \in \mathbf{N}$ such that $\prod_{k=1}^{n}\left(q_{k}+1\right), \prod_{k=1}^{n}\left(q_{k}-1\right)$ and $\prod_{k=1}^{n}\left(q_{k}-2\right)$ are divisible by $p$.

We would like to define a simple ordered Bratteli diagram $(V, E, \leq)$ ([HPS $])$. Let the vertex set $V_{n}$ be $\left\{v_{n}, v_{n}^{\prime}\right\}$ and define the edge set $E_{n}$ so that the connecting matrix from $\mathbf{Z}^{V_{n}}$ to $\mathbf{Z}^{V_{n+1}}$ equals

$$
A_{n}=\left[\begin{array}{cc}
q_{n} & 1 \\
1 & q_{n}
\end{array}\right] \text {. }
$$

Let the source vertex of the initial edge in $r^{-1}\left(v_{n+1}\right)$ and $r^{-1}\left(v_{n+1}^{\prime}\right)$ be $v_{n}$, and the source vertex of the final edge be $v_{n}^{\prime}$. Then, we obtain a simple ordered Bratteli diagram. Let $(X, \phi)$ be the Bratteli-Vershik system of $(V, E, \leq)$. The dimension group $K^{0}(X, \phi)$ is isomorphic to $\mathbf{Q}^{2}$ and the positive cone is

$$
K^{0}(X, \phi)^{+}=\{(x, y) ; \alpha x>|y|\} \cup\{(0,0)\},
$$

and so there are two ergodic measures. The distinguished order unit [1] is equal to $(2,0)$.

Let us construct two extensions of $(X, \phi)$, namely $\left(Y_{0}, \psi_{0}\right)$ and $\left(Y_{1}, \psi_{1}\right)$. We would like to define two simple ordered Bratteli diagrams $\left(W_{0}, F_{0}, \leq\right)$ and $\left(W_{1}, F_{1}, \leq\right)$. Set the vertex set $W_{0, n}$ be $\left\{w_{0, n}, w_{0, n}^{\prime}, w_{0, n}^{\prime \prime}\right\}$ and the vertex set $W_{1, n}$ be $\left\{w_{1, n}, w_{1, n}^{\prime}, w_{1, n}^{\prime \prime}\right\}$. To describe the partial order on the edge set, for each vertex $w$, we denote by $\theta(w)$ the ordered list of the source vertices of edges in $r^{-1}(w)$. Define the partial order on $F_{0}$ by

$$
\begin{aligned}
& \theta\left(w_{0, n+1}\right)=(w_{0, n}, \overbrace{w_{0, n}, \cdots, w_{0, n}}^{q_{n}-2}, w_{0, n}^{\prime \prime}, w_{0, n}^{\prime}), \\
& \theta\left(w_{0, n+1}^{\prime}\right)=(w_{0, n}, \overbrace{w_{0, n}^{\prime}, \cdots, w_{0, n}^{\prime}}^{q_{n}-2}, w_{0, n}^{\prime}, w_{0, n}^{\prime}),
\end{aligned}
$$

and

$$
\theta\left(w_{0, n+1}^{\prime \prime}\right)=(w_{0, n}, \overbrace{w_{0, n}^{\prime \prime}, \cdots, w_{0, n}^{\prime \prime}}^{q_{n}-2}, w_{0, n}^{\prime \prime}, w_{0, n}^{\prime}) .
$$


Similarly, define the partial order on $F_{1}$ by

$$
\begin{aligned}
& \theta\left(w_{1, n+1}\right)=(w_{1, n}, \overbrace{w_{1, n}, \cdots, w_{1, n}}^{q_{n}-2}, w_{1, n}, w_{1, n}^{\prime}), \\
& \theta\left(w_{1, n+1}^{\prime}\right)=(w_{1, n}, \overbrace{w_{1, n}^{\prime}, \cdots, w_{1, n}^{\prime}}^{q_{n}-2}, w_{1, n}^{\prime \prime}, w_{1, n}^{\prime}),
\end{aligned}
$$

and

$$
\theta\left(w_{1, n+1}^{\prime \prime}\right)=(w_{1, n}, \overbrace{w_{1, n}^{\prime \prime}, \cdots, w_{1, n}^{\prime \prime}}^{q_{n}-2}, w_{1, n}^{\prime \prime}, w_{1, n}^{\prime}),
$$

for each $n \in \mathbf{N}$. Then, we get two Cantor minimal systems $\left(Y_{0}, \psi_{0}\right)$ and $\left(Y_{1}, \psi_{1}\right)$ associated with simple ordered Bratteli diagrams $\left(W_{0}, F_{0}, \leq\right)$ and $\left(W_{1}, F_{1}, \leq\right)$. There exists a factor map $\pi_{0}:\left(Y_{0}, \psi_{0}\right) \rightarrow(X, \phi)$ which sends the tower $w_{0, n}$ and $w_{0, n}^{\prime \prime}$ to $v_{n}$, and the tower $w_{0, n}^{\prime}$ to $v_{n}^{\prime}$. In the same way, there exists a factor map $\pi_{1}:\left(Y_{1}, \psi_{1}\right) \rightarrow(X, \phi)$. For $i=0,1$, we set

$$
E_{i}=\left\{x \in X ; \# \pi_{i}^{-1}(x) \neq 1\right\} .
$$

It is not hard to see that $E_{0}$ and $E_{1}$ are disjoint.

Let $\beta$ be the limit of $\prod_{k=1}^{n}\left(q_{k}-2\right) /\left(q_{k}-1\right)$. The dimension group $K^{0}\left(Y_{i}, \psi_{i}\right)$ is isomorphic to $\mathbf{Q}^{3}$ and the positive cone is

$$
K^{0}\left(Y_{i}, \psi_{i}\right)^{+}=\left\{\begin{array}{c}
\alpha a-b-\beta c>0 \\
(a, b, c) ; \\
\alpha a+b+2 \beta c>0 \\
\alpha a+b-\beta c>0
\end{array}\right\} \cup\{(0,0,0)\},
$$

for both $i=0,1$, hence, $\left(Y_{i}, \psi_{i}\right)$ has three ergodic measures. The order embeddings $\pi_{0}^{*}$ and $\pi_{1}^{*}$ send $(x, y) \in \mathbf{Q}^{2}$ to $(x, y, 0)$ and $(x,-y, 0)$, respectively.

Choose $x \in X \backslash\left(E_{0} \cup E_{1}\right)$ and $y_{i} \in Y_{i}$ such that $\pi_{i}\left(y_{i}\right)=x$. The topological joining $(Z, \tau)$ generated by $\left(y_{0}, y_{1}\right)$ is a Cantor minimal system. The dimension group $K^{0}(Z, \tau)$ is isomorphic to the relative direct sum of $K^{0}\left(Y_{0}, \psi_{0}\right)$ and $K^{0}\left(Y_{1}, \psi_{1}\right)$ with respect to $K^{0}(X, \phi)$. Therefore, $K^{0}(Z, \tau)$ equals $\mathbf{Q}^{4}$ and the positive cone is determined by four linear inequalities. In this case, $K^{0}(Z, \tau)$ satisfies the Riesz interpolation property, although neither $\pi_{0}\left(K^{0}(X, \phi)\right)$ nor $\pi_{1}\left(K^{0}(X, \phi)\right)$ is order dense.

\section{Non-coalescence and dimension groups.}

Let $\alpha$ and $\beta$ be two irrational numbers which are linearly independent over $\mathbf{Q}$. By cutting the circle $\mathbf{S}^{1} \cong \mathbf{R} / \mathbf{Z}$ at the points $n \alpha-m \beta$ for $n \in \mathbf{Z}$ and $m \in \mathbf{N} \cup\{0\}$, a Cantor set $Y$ is obtained. Then, the $\alpha$-rotation on $Y$ gives a minimal homeomorphism $\psi$, and the system $(Y, \psi)$ is called the Denjoy system ([PSS $])$. It is easy to see that the $\beta$-rotation induces a non-invertible endomorphism $\gamma$ on $(Y, \psi)$. The dimension group $K^{0}(Y, \psi)$ is isomorphic to the countable direct sum of $\mathbf{Z}$, and $\bmod (\gamma)$ is an endomorphism on it. 
In this section, we would like to construct non-invertible endomorphisms of Cantor minimal systems which induce automorphisms on the dimension groups. We say an automorphism $\gamma$ of a Cantor minimal system $(X, \phi)$ has essentially infinite order, if $\gamma^{i}$ does not equal $\phi^{j}$ for all $i \in \mathbf{N}$ and $j \in \mathbf{Z}$.

Lemma 9. Let $(X, \phi)$ be a Cantor minimal system, $\gamma \in C(\phi)$ be an automorphism which has essentially infinite order and $x_{\max } \in X$ be a point. Then, there exist a Cantor minimal system $(Y, \psi)$ and a factor map $\pi$ : $(Y, \psi) \rightarrow(X, \phi)$ such that the following properties are satisfied:

(i) $\pi^{*}$ is an order isomorphism between $K^{0}(X, \phi)$ and $K^{0}(Y, \psi)$.

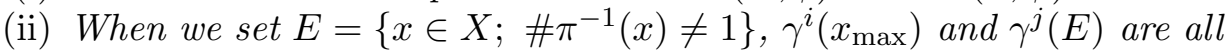
disjoint for $i, j \in \mathbf{Z}$.

Proof. We may assume that $X$ is the infinite path space of an ordered Bratteli diagram $(V, E, \leq), x_{\max }$ is the unique maximal path and $\phi$ is the BratteliVershik map. Let $x_{\min }$ be the unique minimal path. We denote the source map by $s: E_{n} \rightarrow V_{n}$ and the range map by $r: E_{n} \rightarrow V_{n+1}$. The symbol $E_{n, m}$ means the set of finite paths from $V_{n}$ to $V_{m}$ and $[e]$ for $e \in E_{n, m}$ means the corresponding clopen set of $X$. Let $A_{n}$ be the incidence matrix from $\mathbf{Z}^{V_{n}}$ to $\mathbf{Z}^{V_{n+1}}$, and $h(v)$ be the number of edges connecting $v_{0} \in V_{0}$ to $v \in V_{n}$.

By telescoping the diagram, we would like to choose $v_{n} \in V_{n}$ and $f_{n}, f_{n}^{\prime} \in$ $E_{n}$ for each $n \in \mathbf{N}$ which satisfy the following:

- The source and range vertex of $f_{n}$ and $f_{n}^{\prime}$ are $v_{n}$ and $v_{n+1}$, respectively.

- The clopen sets $\gamma^{j}\left(\left[f_{n}\right]\right)$ and $\gamma^{k}\left(\left[f_{n}^{\prime}\right]\right)$ for $|j|,|k| \leq n$ are all disjoint.

- The clopen set $\left[f_{n}\right] \cup\left[f_{n}^{\prime}\right]$ doesn't contain $\gamma^{k}\left(x_{\max }\right)$ and $\gamma^{k}\left(x_{\min }\right)$ for $|k| \leq n$.

The construction of $v_{n}, f_{n}$ and $f_{n}^{\prime}$ is by induction. At first, we choose $v_{1} \in V_{1}$ arbitrarily. Let us assume that $v_{n}, f_{n-1}$ and $f_{n-1}^{\prime}$ have been already chosen. Since $\gamma$ has essentially infinite order, we can find $m>n$ and $v^{\prime} \in V_{m}$ satisfying

$$
[e] \cap \gamma^{k}[e] \text { is empty for all } e \in E_{v_{n}, v^{\prime}}, 0<|k| \leq n
$$

and

$$
\# E_{v_{n}, v^{\prime}}>2(2 n+1)+h\left(v_{n}\right)(4 n+1)+1,
$$

where $E_{v_{n}, v^{\prime}}$ means the set of edges from $v_{n}$ to $v^{\prime}$. Choose $f \in E_{v_{n}, v^{\prime}}$ so that $[f]$ does not contain $\gamma^{k}\left(x_{\max }\right)$ and $\gamma^{k}\left(x_{\min }\right)$ for $|k| \leq n$. For a large number $l>m$, we may assume that

$$
\forall e \in E_{0, l}, \forall|k| \leq 2 n, \quad \exists e^{\prime} \in E_{n, m}, \quad \text { s.t. } \gamma^{k}([e]) \subset\left[e^{\prime}\right] .
$$

Then, let $f_{n} \in E_{n, l}$ be an arbitrary extension of $f$ and choose $f_{n}^{\prime} \in E$ as an extension of an edge of $E_{v_{n}, v^{\prime}}$ so that the above property is satisfied. Hence, the induction is completed. 
Let $F$ be the set of infinite paths consisting of $f_{n}$ 's and $f_{n}^{\prime}$ 's and $E$ be the union of all $\phi^{k}(F)$ for $k \in \mathbf{Z}$. We construct a new ordered Bratteli diagram $\left(W, E^{\prime}, \leq\right)$. Define the vertex set $W_{n}$ as the disjoint union of $V_{n}$ and $v_{n}^{\prime}$, and let the map $\rho: W_{n} \rightarrow V_{n}$ be $\rho\left(v_{n}^{\prime}\right)=v_{n}$ and $\rho(v)=v$ for $v \in V_{n}$. Define the edge set $E_{n}^{\prime}$ so that the incidence matrix $B_{n}$ satisfies

$$
B_{n}\left(w, w^{\prime}\right)=\left\{\begin{array}{cl}
0 & w=v_{n}^{\prime}, w^{\prime} \neq v_{n+1}, v_{n+1}^{\prime} \\
1 & w=v_{n}^{\prime}, w^{\prime}=v_{n+1}, v_{n+1}^{\prime} \\
A_{n}\left(v_{n}, v_{n+1}\right)-1 & w=v_{n}, w^{\prime}=v_{n+1}, v_{n+1}^{\prime} \\
A_{n}\left(\rho(w), \rho\left(w^{\prime}\right)\right) & \text { otherwise }
\end{array}\right.
$$

for $w \in W_{n}$ and $w^{\prime} \in W_{n+1}$. It is not hard to check that the inductive limit of $\left(\mathbf{Z}^{W_{n}}\right)_{n}$ with the connecting maps $\left(B_{n}\right)_{n}$ is order isomorphic to $K^{0}(X, \phi)$. Let us define the linear order on the set $r^{-1}\left(w^{\prime}\right)$ for each $w^{\prime} \in W_{n+1}$. When $w^{\prime}$ is not equal to $v_{n+1}$ nor $v_{n+1}^{\prime}$, we define the linear order by exactly the same way as in $r^{-1}\left(\rho\left(w^{\prime}\right)\right)$. If $w^{\prime}$ is $v_{n+1}$ or $v_{n+1}^{\prime}$, we change the source vertex of the edge $f_{n}$ or $f_{n}^{\prime}$, respectively, to $v_{n}^{\prime}$. Then, we get a well-defined ordered Bratteli diagram.

Let $(Y, \psi)$ be the Cantor minimal system determined by $\left(W, E^{\prime}, \leq\right)$. Obviously, there is a factor map $\pi:(Y, \psi) \rightarrow(X, \phi)$, and $\pi$ is not one-to-one exactly on the subset $E$. By the construction of $E$, we see that $E$ satisfies the condition (ii) above.

For an automorphism $\gamma$ which induces the identity on the dimension group, we introduced a new invariant $\eta(\gamma)$ in the Ext group $([\mathbf{M}])$. When $\gamma$ is a non-invertible endomorphism, we can define $\eta(\gamma)$ in the same way, by replacing $\gamma^{-1}$ with $\gamma$ in $[\mathbf{M}]$.

Theorem 10. Let $(X, \phi)$ be a Cantor minimal system, $\gamma \in C(\phi)$ be an automorphism which has essentially infinite order. Then, there exists a Cantor minimal system $(Z, \tau)$, a factor map $\Phi:(Z, \tau) \rightarrow(X, \phi)$ and a non-invertible endomorphism $\widetilde{\gamma} \in C(\tau)$ such that $\gamma \circ \Phi=\Phi \circ \widetilde{\gamma}$ and $\Phi$ induces the isomorphism between the dimension groups $K^{0}(X, \phi)$ and $K^{0}(Z, \tau)$. Moreover, when $\bmod (\gamma)$ is the identity, $\eta(\widetilde{\gamma})$ equals $\eta(\gamma)$ in the Ext group.

Proof. Choose $x_{\max } \in X$ arbitrarily and let $(Y, \psi)$ and $\pi$ as in Lemma 9. For each $i \in \mathbf{N} \cup\{0\}$, let $y_{i} \in Y$ be the unique point such that $\pi\left(y_{i}\right)=$ $\gamma^{i}\left(x_{\max }\right)$ and $(Z, \tau)$ be the topological joining generated by $\left(y_{i}\right)_{i}$. By applying Lemma 1 to $\gamma^{-i} \circ \pi$, we see that $(Z, \tau)$ is a Cantor minimal system. Let $\Phi$ be the composition of $\pi$ and the projection from $Z$ to the first summand. By Corollary 8, the factor map $\Phi$ induces an isomorphism between $K^{0}(X, \phi)$ and $K^{0}(Z, \tau)$. It is easily seen that the one-sided subshift on $Y^{\mathbf{N} \cup\{0\}}$ gives the well-defined centralizer $\widetilde{\gamma} \in C(\tau)$ and $\widetilde{\gamma}$ is a non-invertible endomorphism satisfying $\gamma \circ \Phi=\Phi \circ \widetilde{\gamma}$. 
By applying the above theorem to an odometer system $(X, \phi)$, we get a Cantor minimal system $(Z, \tau)$ which is strong orbit equivalent to $(X, \phi)$ and has a non-invertible endomorphism $\gamma$. Of course, $\gamma$ induces the identity map on the dimension group $K^{0}(Z, \tau)$. Actually, in the proof of Lemma 9, we can find the Cantor minimal system $(Y, \psi)$ in the class of Toeplitz subshifts, if $(X, \phi)$ is an odometer system. Therefore, the Cantor minimal system $(Z, \tau)$ is the projective limit of Toeplitz minimal subshifts. But, the system $(Z, \tau)$ itself is not expansive. In [D], T. Downarowicz constructed an example of a Toeplitz minimal subshift which admits a non-invertible endomorphism. We can check that the endomorphism in his example does not induce the identity on the dimension group. The author doesn't know non-coalescent minimal subshifts except for Downarowicz's example.

Acknowledgments. The author is grateful to Professor Izumi for his constant encouragement.

\section{References}

[D] T. Downarowicz, The royal couple conceals their mutual relationship: A noncoalescent Toeplitz flow, Israel J. Math., 97 (1997), 239-251, MR 99d:28031, Zbl 0893.54032.

[GPS] T. Giordano, I.F. Putnam and C.F. Skau, Topological orbit equivalence and $C^{*}$ crossed products, J. Reine Angew. Math., 469 (1995), 51-111, MR 97g:46085, Zbl 0834.46053.

[GPS2] _ K-theory and asymptotic index for certain almost one-to-one factors, Math. Scand., 89(2) (2001), 297-319, CMP 1868179.

[GH] K.R. Goodearl and D.E. Handelman, Metric completions of partially ordered abelian groups, Indiana. Univ. Math. J., 29 (1980), 861-895, MR 82b:06020, Zbl 0455.06012 .

[HPS] R.H. Herman, I.F. Putnam and C.F. Skau, Ordered Bratteli diagrams, dimension groups and topological dynamics, Internat. J. Math., 3 (1992), 827-864, MR 94f:46096, Zbl 0786.46053.

[HR] E. Hewitt and A.R. Ross, Abstract Harmonic Analysis, Volume I, Grundlehren der Matematischen Wissenschaften, 115, Springer-Verlag, 1979, MR 81k:43001, Zbl 0416.43001.

[M] H. Matui, Ext and OrderExt classes of certain automorphisms of $C^{*}$-algebras arising from Cantor minimal systems, Canad. J. Math., 53 (2001), 325-354, CMP 1820 912, Zbl 0968.46046.

[M2] , Finite order automorphisms and dimension groups of Cantor minimal systems, J. Math. Soc. Japan, 54(1) (2002), 135-160, CMP 1864931.

[PSS] I.F. Putnam, K. Schmidt and C.F. Skau, $C^{*}$-algebras associated with Denjoy homeomorphisms of the circle, J. Operator Theory, 16 (1986), 99-126, MR 87j:46126, Zbl 0611.46067. 
[R] D. Rudolph, An example of a measure-preserving map with minimal self-joinings, J. Analyse Math., 35 (1979), 97-122, MR 81e:28011, Zbl 0446.28018.

Received June 10, 2000 and revised June 26, 2001.

Department of Mathematics and Informations

Chiba University

YAYOITYO 1-33, INAGEKU

CHIBA 263-8522

JAPAN

E-mail address: matui@math.s.chiba-u.ac.jp 